\title{
Optimizing Nanostructure Size to Yield High Raman Signal Enhancement by Electron Energy Loss Spectroscopy
}

\author{
Yitian Zeng $^{1 *}$, Steven Madsen ${ }^{1}$, Andrew Yankovich ${ }^{2}$, Eva Olsson $^{2}$ and Robert Sinclair ${ }^{1}$ \\ 1. Department of Materials Science and Engineering, Stanford University, Stanford, United States \\ 2. Department of Physics, Chalmers University of Technology, Gothenburg, Sweden \\ *Corresponding author: ytzeng1@stanford.edu
}

The Raman signal from an organic molecule can be significantly enhanced by its contact with a noble metal surface. This phenomenon, known as surface enhanced Raman spectroscopy (SERS) in biological and chemical sensing applications, is strongly related to the localized surface plasmon resonance (LSPR) of the nanostructures [1]. Here we use electron energy loss spectroscopy (EELS) to study the relationship between nanoparticle structure and LSPR, and the overall effect on Raman signal enhancement.

Nanodisc arrays of gold with diameters of $150 \mathrm{~nm}, 90 \mathrm{~nm}, 75 \mathrm{~nm}$ and $55 \mathrm{~nm}$ were fabricated directly on $\mathrm{Si}_{3} \mathrm{~N}_{4}$ membrane TEM grids using electron beam lithography (Fig. 1) [2]. EELS spectrum images were collected around four discs in each array (inset of Fig. 2a) using a monochromated scanning transmission electron microscope (mono-STEM) operated at $300 \mathrm{kV}$. For each individual nanodisc of $150 \mathrm{~nm}$ in diameter, the EELS spectrum close to the edges (black) differs from that in the disc center (green). The EELS intensity map at electron energy loss of $1.7 \mathrm{eV}$ (Fig. 2b) shows that the strongest surface plasmon mode is located closely to the nanodisc edges. A dark mode, manifested as an additional peak at $2.3 \mathrm{eV}$, resides primarily in the center of nanodiscs (Fig. 2c) [2].

Fig. 3a shows EEL spectra around edges of different size nanodiscs and the 638nm laser energy (1.9eV) used for the Raman excitation. In order of increasing LSPR energy, we found $1.7 \mathrm{eV}$ for $150 \mathrm{~nm}$ nanodiscs, $1.9 \mathrm{eV}$ for $90 \mathrm{~nm}$ nanodiscs, $2.0 \mathrm{eV}$ for $75 \mathrm{~nm}$ nanodiscs, and $2.1 \mathrm{eV}$ for $55 \mathrm{~nm}$ nanodiscs. This red shift of surface plasmon energy as nanodiscs size increases is also observed in various optical and simulation studies $[3,4]$. The same samples coated with Rhodamine $6 \mathrm{G}$ were subsequently illuminated with a $638 \mathrm{~nm}$ laser to collect Raman spectra (Fig. 3b) using a Horiba XploRA+ confocal Raman microscope. We observed that when the excitation laser energy is very close to LSPR (as in the case of 90nm nanodisc array for which the mismatch is less than 5\%), the Raman signal experiences a significant enhancement compared with off-resonance situations. This observation strongly supports an electromagnetic enhancement mechanism [2]. A damped harmonic oscillator model is implemented to estimate Raman intensity in Fig. 3c and shows reasonable agreement with experimentally measured values.

This study demonstrates that surface plasmon response can be finely tuned to match the excitation laser energy by controlling nanostructure size to achieve optimum Raman signal enhancement. It will serve as a useful tool to engineer nanoparticles for cancer diagnostics and treatment [5].

References:

[1] R. Alvarez-Puebla, J. Phys. Chem. Lett. 3 (2012), p. 857-866.

[2] S.J. Madsen et al. ACS Photonics 4 (2017), p.268-274. 
[3] S.A. Maier in "Plasmonics: Fundamentals and Applications". (Springer US, New York) p.66-88.

[4] G.D. Bernasconi et al. ACS Photonics 4 (2017), p.156-164.

[5] M.F. Kircher et al. Nature Medicine 18 (2012), p.829-834.

[6] This work was supported by the Center for Cancer Nanotechnology Excellence for Translational Diagnostics (CCNE-TD) at Stanford University through an award (grant no: U54 CA199075) from the National Cancer Institute (NCI) of the National Institutes of Health (NIH).

(a)

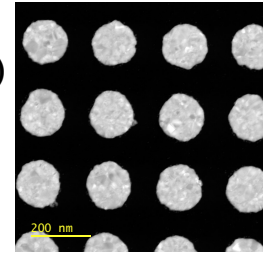

(b)

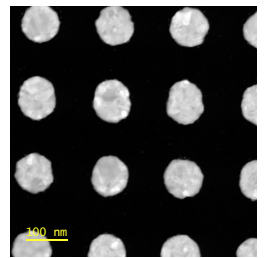

(c)

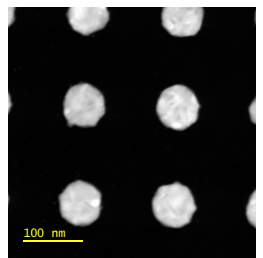

(d)



Figure 1. Annular dark field images of nanodisc arrays with the same edge to edge spacing of $90 \mathrm{~nm}$ and diameters of (a) $150 \mathrm{~nm}$, (b) $90 \mathrm{~nm}$, (c) $75 \mathrm{~nm}$ and (d) $55 \mathrm{~nm}$.

(a)

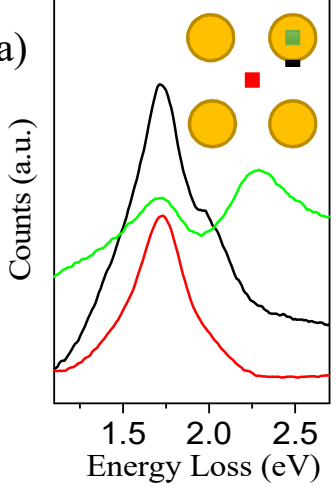

(b)

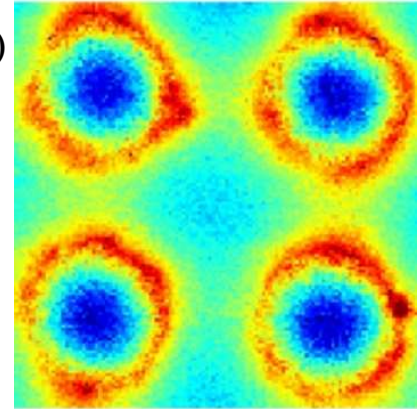

Energy $=1.7 \mathrm{eV}$

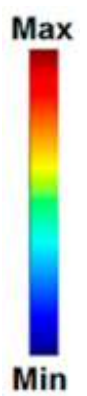

Min (c)

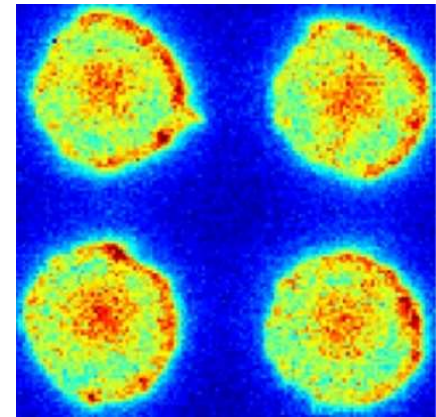

Energy $=2.3 \mathrm{eV}$

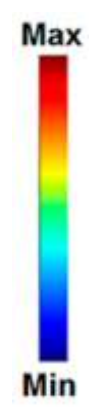

Min

Figure 2. (a) EELS spectra from the 150nm diameter array at the red, black and green boxed regions. (b) EELS intensity map of $150 \mathrm{~nm}$ disc array at energy loss of $1.7 \mathrm{eV}$ showing strongest surface plasmon modes located near edges and (c) at energy loss of 2.3eV showing dark modes in the disc centers [2].

(a)

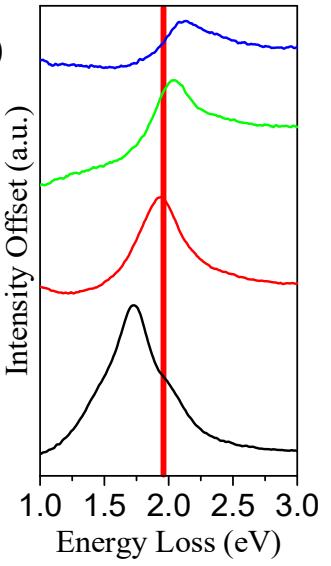

(b)

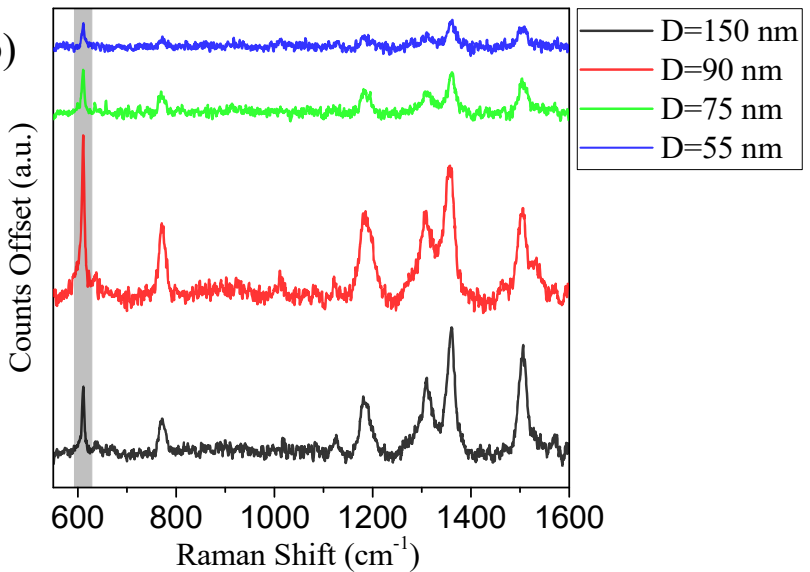

(c)

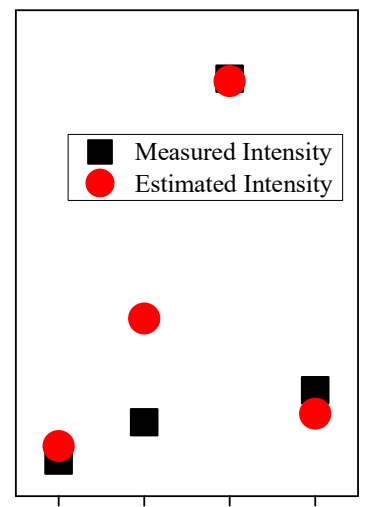

$55 \mathrm{~nm} 75 \mathrm{~nm} 90 \mathrm{~nm} 150 \mathrm{~nm}$ Disk Diameter

Figure 3. (a) EEL spectra around edges of different size nanodiscs after zero loss peak subtraction showing surface plasmon peaks. The red vertical line represents the $638 \mathrm{~nm}$ laser energy. (b) Raman spectra measured with $638 \mathrm{~nm}$ laser. The $90 \mathrm{~nm}$ disc array exhibits strongest Raman signal for the 611 $\mathrm{cm}^{-1}$ peak. (c) Estimated intensity from EELS based on damped oscillator model and measured Raman integrated peak intensity at $611 \mathrm{~cm}^{-1}$. 